\title{
Active nematic gels as active relaxing solids
}

\author{
Stefano S. Turzi ${ }^{*}$ \\ Dipartimento di Matematica, Politecnico di Milano, Piazza Leonardo da Vinci 32, 20133 Milano, Italy
}

(Received 11 September 2017; published 9 November 2017)

\begin{abstract}
I propose a continuum theory for active nematic gels, defined as fluids or suspensions of orientable rodlike objects endowed with active dynamics, that is based on symmetry arguments and compatibility with thermodynamics. The starting point is our recent theory that models (passive) nematic liquid crystals as relaxing nematic elastomers. The interplay between viscoelastic response and active dynamics of the microscopic constituents is naturally taken into account. By contrast with standard theories, activity is not introduced as an additional term of the stress tensor, but it is added as an external remodeling force that competes with the passive relaxation dynamics and drags the system out of equilibrium. In a simple one-dimensional channel geometry, we show that the interaction between nonuniform nematic order and activity results in either a spontaneous flow of particles or a self-organization into subchannels flowing in opposite directions.
\end{abstract}

DOI: 10.1103/PhysRevE.96.052603

\section{INTRODUCTION}

The mathematical modeling of biophysical and active materials poses new theoretical challenges for their unique features. Examples of active matter comprise bacterial swarms [1], the cellular cytoskeleton [2,3], and in vitro cell extracts. Nonbiological examples include vibrated granular material [4]. These systems have attracted much interest in recent years both from a purely theoretical perspective and for their potential applications. They are characterized by a strong deviation from thermal equilibrium due to the environmental energy supply and the active dynamics of the system's microscopic subunits. The simplest, yet successful, theoretical description of active matter is based on continuum models for single-component suspensions of rodlike objects. These models have been originally developed to describe (passive) nematic liquid crystals, e.g., Ericksen-Leslie theory. The key features of an active system, namely, its viscoelastic response $[3,5,6]$ and the active dynamics due to energy consumption of the material subunits, are usually added in an ad hoc manner to the passive physical model. Viscoelasticity is taken into account by postulating a Maxwell relaxation time and activity is usually introduced by assuming an active stress, proportional to the nematic ordering tensor $\mathbf{Q}$. In its simplest form, the active stress is postulated to be of the form $\mathbf{T}_{\zeta}=-\zeta \mathbf{Q}$, where $\zeta$ is a modeling coefficient that measures the strength of activity. When $\zeta>0$ the material is extensile, while if $\zeta<0$ it has a natural tendency to contract. This contribution to the stress tensor has been deduced on microscopic grounds by Simha and Ramaswamy [7], and it has been widely used since then (see, for instance, Refs. [2-4,6,8]). The equations that emerge are those proposed by Simha and Ramaswamy [7] for selfpropelling organisms, but similar models have been developed in the context of the cytoskeleton of living cells, a network of polar actin filaments, made active by molecular motors that consume ATP $[2,3]$. A multicomponent theory based on irreversible thermodynamics is derived in $[2,9,10]$. The theory seems to be able to make a number of successful predictions, e.g., the onset of spontaneous flow [11,12], motility, and spontaneous division of active nematic droplets [13-15].

\footnotetext{
*stefano.turzi@polimi.it
}

However, I believe that the assumption of an active stress does not correctly capture the true essential nature of an active behavior. The responses of tissues to elastic forces are quite different from the passive mechanical properties of composite materials. For example, on short time scales the passive elastic response of the matrix and the cellular cytoskeleton dominate the mechanical response of the tissue; on longer time scales many cell types (such as muscle cells, fibroblasts, endothelial cells) can reorganize to reduce their internal stress and thus reach a relaxed or natural state. Cross-links between polymer filaments define a natural distance; in other words, they define a natural metric in the material that I shall call shape tensor. A strained viscoelastic material has a natural tendency to recover this natural state and can reconfigure its internal structure to perform this relaxation in an efficient manner (e.g. viscous relaxation).

By contrast, activity is an external remodeling force that competes with the passive remodeling and may drive the microscopic reorganization away from the natural metric. The remodeling forces model the interaction with the chemical fuel, and tension is generated as a consequence of the crawling motion of aligned filaments. Hence, I believe that activity is best described by a term in the evolution equation of the internal structure of the material, rather than directly in the stress tensor. Indeed, chemical fuel is consumed, and hence the power exerted by the active term should be nonzero, even in the absence of any macroscopic flow. This argument contrasts with the introduction of the active term $\mathbf{T}_{\zeta}$ in the Cauchy stress tensor: if the macroscopic flow, $\mathbf{v}$, is zero, the active stress power, calculated as $\mathbf{T}_{\zeta} \cdot \nabla \mathbf{v}$, must also vanish.

It is worth noticing that, since the actual macroscopic response of the material is related to the "distance" between its present state and its relaxed state, activity has an indirect contribution to the stress tensor as the effective material response depends on it. I will make these statements mathematically more precise in the next sections.

A similar situation occurs in the continuum theory for growth and remodeling of solidlike biological tissues, e.g., muscles [16-22]. Some theories use a remodeling force and an active strain (instead of an active stress) to model the ability of the muscle to actively modify its natural metric. The remodeling is then performed, via a remodeling force, at the expense of a chemical fuel. Chemical energy must be 
supplied also in the absence of any macroscopic motion-for example, during an isometric exercise-as it is needed for the remodeling of the muscle internal structure. Hence, to calculate the stress power, the active term has to be paired with a kinematic quantity related to the material remodeling rather than with the macroscopic velocity gradient $\nabla \mathbf{v}$.

An active stress of the form $\mathbf{T}_{\zeta}=-\zeta \mathbf{Q}$ has also been criticized by Brand et al. [23] on the ground of compatibility with linear irreversible thermodynamics. Their argument is related to the fact that reversible and dissipative forces behave differently under time reversal: the dissipative forces have the same signature as their conjugate fluxes under time reversal, while reversible forces have the opposite signature. Time reversal implies a change of sign of the velocity field $\mathbf{v}$. In our case, the active stress is coupled with the gradient of velocity $\nabla \mathbf{v}$ and its contribution to the entropy production is $-\zeta \mathbf{Q} \cdot \nabla \mathbf{v}$. If $\mathbf{T}_{\zeta}$ has to be irreversible, then the activity coefficient $\zeta$ must also change sign under time reversal, which is a rather unusual behavior for a scalar.

In the present paper we propose a simple and systematic derivation of the continuum equations of viscoelastic active nematic gels that is based on symmetry arguments, relaxation dynamics, and compatibility with linear irreversible thermodynamics. For simplicity, we discuss only a one-component active gel considering therefore that the complex composition of active materials such as the cytoskeleton can be described by an effective single-component fluid. More complicated theories that employ a multicomponent description of the active fluid and use linear irreversible thermodynamics are to be found in Refs. [9,10].

The theory is developed in Sec. II. Section III describes the hydrodynamic approximation, and Sec. IV deals with some simple applications of the theory.

\section{SHAPE TENSOR AND RELAXATION DYNAMICS}

Since continuum theories are essentially based on symmetry arguments and general physical principles, they are generally applicable to a whole range of physical systems. On the downside, they do not involve significant microscopic considerations and the material parameters are purely phenomenological. In the present section we put forward a continuum theory for nematic active gels. The viscoelastic passive response is derived by relaxing the elastic response of a nematic liquid-crystal elastomer. The shape tensor in this theory plays the role of a metric tensor and describes, on a macroscopic ground, the information about the equilibrium distances among the centers of mass of the microscopic constituents.

\section{A. Internal and external degrees of freedom}

The first key idea is to study, separately, the degrees of freedom associated with the elastic deformations and the "microscopic" degrees of freedom related to the material relaxation and reorganization. The first process is reversible and conserves the energy, while the second process involves material reorganization and is irreversible. I assume that activity directly interferes with the internal degrees of freedom and competes with the natural tendency of the material to reach the equilibrium state. To this end, we introduce the Kröner-Lee-
Rodriguez multiplicative decomposition for the deformation gradient $\mathbf{F}=\mathbf{F}_{\mathrm{e}} \mathbf{G}$ [24-26]. For later convenience we also define the inverse relaxing strain $\mathbf{H}=\left(\mathbf{G}^{T} \mathbf{G}\right)^{-1}$, so that the effective left-Cauchy-Green deformation tensor can be written as

$$
\mathbf{B}_{\mathrm{e}}=\mathbf{F}_{\mathrm{e}} \mathbf{F}_{\mathrm{e}}^{T}=\mathbf{F G}^{-1} \mathbf{G}^{-T} \mathbf{F}^{T}=\mathbf{F} \mathbf{H} \mathbf{F}^{T} \text {. }
$$

The same decomposition has been recently applied to explain the hints of viscoelasticity that remain at the hydrodynamic level when a sound wave propagates inside a nematic crystal [27-30].

The tensor $\mathbf{G}$ models the microscopic remodeling of the material. In other words, it describes the inelastic dissipative (irreversible) processes within the material. By contrast, $\mathbf{F}_{\mathrm{e}}$ is related to the elastic (reversible) response.

\section{B. Shape tensor and free energy}

Contrary to most hydrodynamic theories of active nematic gels I do not use the ordering tensor $\mathbf{Q}$ to take into account uniaxial nematic symmetry of the microscopic subunits. I rather introduce a uniaxial, unit determinant, shape tensor, common to the theory of nematic elastomers

$$
\boldsymbol{\Psi}(\varrho, \mathbf{n})=a(\varrho)^{2}(\mathbf{n} \otimes \mathbf{n})+a(\varrho)^{-1}(\mathbf{I}-\mathbf{n} \otimes \mathbf{n}),
$$

where $a(\varrho)$ is a (density-dependent) shape parameter and the preferred direction $\mathbf{n}$ lives in the actual configuration of the body since it is not materially linked to body deformations. The shape tensor is spherical, prolate, or oblate, respectively, for $a(\varrho)=1, a(\varrho)>1$, or $a(\varrho)<1$. The material parameter $a(\varrho)$ gives the amount of spontaneous elongation along $\mathbf{n}$ in a uniaxially ordered phase. It is a combined measure of the degree of order and of the strength of the nematicelastic coupling. The tensor $\boldsymbol{\Psi}$ represents a volume-preserving uniaxial stretch along the current direction of the director $\mathbf{n}$. In particular, the unit determinant assumption implies that growth is not taken into account in the model.

It must be noted that the same tensor is used to describe the coupling between strain and orientation in nematic elastomers, where $\boldsymbol{\Psi}$ is usually interpreted as a effective step-length tensor that reflects the current nematic ordering in the polymer network [31]. In our model, $\boldsymbol{\Psi}$ represents the spontaneous metric tensor that is dictated by the coarse-grained anisotropy of the subunits. The equilibrium configuration of the subunits is usually anisotropic in the direction of $\mathbf{n}$ and the measure of this anisotropy is yielded by the value of $a(\varrho)$.

For fast relaxation times, only the local form of the elastic energy is important, so we do not need to specify its exact expression globally. However, in this context it is natural to assume the standard energy of polymer physics, i.e., neo-Hookean elasticity. In particular, given the uniaxial symmetry of the constituents, I posit that the elastic response is governed by the nematic elastomer free energy [31], written in terms of $\mathbf{F}_{\mathrm{e}}$. Hence, I posit the following free-energy density per unit mass:

$$
\begin{aligned}
\sigma\left(\varrho, \mathbf{B}_{\mathrm{e}}, \mathbf{n}, \nabla \mathbf{n}\right)= & \sigma_{0}(\varrho)+\frac{1}{2} \mu\left[\operatorname{tr}\left(\boldsymbol{\Psi}^{-1} \mathbf{B}_{\mathrm{e}}-\mathbf{I}\right)\right. \\
& \left.-\log \operatorname{det}\left(\boldsymbol{\Psi}^{-1} \mathbf{B}_{\mathrm{e}}\right)\right]+\sigma_{\mathrm{Fr}}(\varrho, \mathbf{n}, \nabla \mathbf{n}),
\end{aligned}
$$

where $\varrho$ is the density, $\varrho \mu$ is the shear modulus, and $\mathbf{I}$ is the identity tensor. I have also introduced the classical 
Oseen-Frank potential $\sigma_{\mathrm{Fr}}(\varrho, \mathbf{n}, \nabla \mathbf{n})$ [32] that favors the alignment of the director field $\mathbf{n}$. The isotropic term, $\sigma_{0}(\varrho)$, takes into account compressibility. It does not depend on $\mathbf{F}_{\mathrm{e}}$ and is thus not affected by stress relaxation. This is related to the fact that stresses do not vanish in a purely isochoric deformation. By contrast, viscoelastic materials relax the shear stress after a sufficiently long time.

\section{Dissipation}

Let $\mathcal{P}_{t}$ be an arbitrary region that convects with the body. We restrict attention to a purely mechanical theory based on the requirement that the temporal increase in kinetic and free energy of $\mathcal{P}_{t}$ be less than or equal to the power expended on $\mathcal{P}_{t}$ by the external forces. The difference being the power dissipated in irreversible processes. Specifically, for any isothermal process, for any portion $\mathcal{P}_{t}$ of the body at all times, we require

$$
\mathcal{D}:=W^{(\mathrm{ext})}-\dot{K}-\dot{\mathcal{F}} \geqslant 0,
$$

where $W^{(\text {ext })}$ is the power expended by the external forces, $\dot{K}$ is the rate of change of the kinetic energy, $\dot{\mathcal{F}}$ is the rate of change of the free energy, and the dissipation $\mathcal{D}$ is a positive quantity that represents the energy loss due to an irreversible process (entropy production). Here, an overdot indicates the material time derivative. More precisely, I define

$$
\begin{aligned}
& W^{(\mathrm{ext})}:= \int_{\mathcal{P}_{t}} \mathbf{b} \cdot \mathbf{v} d v+\int_{\partial \mathcal{P}_{t}} \mathbf{t}_{(\boldsymbol{v})} \cdot \mathbf{v} d a+\int_{\mathcal{P}_{t}} \mathbf{g} \cdot \dot{\mathbf{n}} d v \\
&+\int_{\partial \mathcal{P}_{t}} \mathbf{m}_{(\boldsymbol{v})} \cdot \dot{\mathbf{n}} d a+\int_{\mathcal{P}_{t}} \mathbf{T}_{\mathrm{a}} \cdot \mathbf{B}_{\mathrm{e}}^{\nabla} d v, \\
& K+\mathcal{F}:= \int_{\mathcal{P}_{t}}\left(\frac{1}{2} \varrho \mathbf{v}^{2}+\varrho \sigma\left(\varrho, \mathbf{B}_{\mathrm{e}}, \mathbf{n}, \nabla \mathbf{n}\right)\right) d v, \quad \xi \geqslant 0, \\
& \mathcal{D}=\int_{\mathcal{P}_{t}} \xi d v, \quad \xi
\end{aligned}
$$

where $\mathbf{v}$ is the velocity field, and

$$
\mathbf{B}_{\mathrm{e}}^{\nabla}:=\left(\mathbf{B}_{\mathrm{e}}\right)^{\cdot}-(\nabla \mathbf{v}) \mathbf{B}_{\mathrm{e}}-\mathbf{B}_{\mathrm{e}}(\nabla \mathbf{v})^{T}=\mathbf{F} \dot{\mathbf{H}} \mathbf{F}^{T}
$$

is the codeformational derivative ${ }^{1}[33,34]$, a frame-indifferent time derivative of $\mathbf{B}_{\mathrm{e}}$ relative to a convected coordinate system that moves and deforms with the flowing body. The unit vector $\boldsymbol{v}$ is the external unit normal to the boundary $\partial \mathcal{P}_{t} ; \mathbf{b}$ is the external body force and $\mathbf{t}_{(v)}$ is the external traction on the bounding surface $\partial \mathcal{P}_{t}$. The vector fields $\mathbf{g}$ and $\mathbf{m}_{(v)}$ are the external generalized forces conjugate to the microstructure: $\mathbf{n} \times \mathbf{g}$ is usually interpreted as "external body moment" and $\mathbf{n} \times \mathbf{m}_{(v)}$ is interpreted as "surface moment per unit area" (the couple stress vector). This interpretation comes from the identity $\dot{\mathbf{n}}=\mathbf{w} \times \mathbf{n}$, where $\mathbf{w}$ is the (local) angular velocity of the director, so that, for instance, the external power density is written as $\mathbf{g} \cdot \dot{\mathbf{n}}=\mathbf{w} \cdot(\mathbf{n} \times \mathbf{g})$.

The last term in Eq. (5) is particularly interesting and new: $\mathbf{T}_{\mathrm{a}}$ is a second-rank tensor that represents an external remodeling force [35], i.e., an external generalized force that

\footnotetext{
${ }^{1}$ Also known as upper-convected time derivative, upper-convected rate, or contravariant rate.
}

competes with the natural microscopic reorganization of the body. It must be noted that $\mathbf{T}_{\mathrm{a}}$ has the same dimensions of a Cauchy stress tensor and is conjugate to the remodeling velocity field $\mathbf{B}_{\mathrm{e}}^{\nabla}$. By contrast, the classical active stress $\mathbf{T}_{\zeta}$ is paired with the macroscopic velocity gradient $\nabla \mathbf{v}$. The possibly unfamiliar time derivative $\mathbf{B}_{\mathrm{e}}^{\nabla}$ has the right properties to represent the kinematics of reorganization: (1) it is frame invariant, (2) it vanishes whenever the deformation is purely elastic and there is no evolution of the natural configuration, ${ }^{2}$ and (3) as shown in the Appendix, it comes out naturally when studying the passive remodeling [see Eq. (A8) of the Appendix, where the material time derivative of $\mathcal{F}$ is explicitly calculated]. The same derivative also appears in the three-dimensional models for Maxwell viscoelastic fluids [33]. Finally, the remodeling power, $\mathbf{T}_{\mathrm{a}} \cdot \mathbf{B}_{\mathrm{e}}^{\nabla}$, depends only on the point value of the internal velocity field $\mathbf{B}_{\mathrm{e}}^{\nabla}$, and not on any of its spatial gradients. Hence, as far as remodeling dynamics is concerned, the present is therefore a theory of grade zero.

After some algebra, reported in the Appendix for ease of reading, the dissipation is recast in the following form:

$$
\begin{aligned}
\mathcal{D}= & \int_{\mathcal{P}_{t}}(\mathbf{b}-\varrho \dot{\mathbf{v}}+\operatorname{div} \mathbf{T}) \cdot \mathbf{v} d v+\int_{\partial \mathcal{P}_{t}}\left(\mathbf{t}_{(\boldsymbol{v})}-\mathbf{T} \boldsymbol{v}\right) \cdot \mathbf{v} d a \\
& +\int_{\mathcal{P}_{t}}(\mathbf{g}-\mathbf{h}) \cdot \dot{\mathbf{n}} d v+\int_{\partial \mathcal{P}_{t}}\left[\mathbf{m}_{(\boldsymbol{v})}-\left(\varrho \frac{\partial \sigma}{\partial \nabla \mathbf{n}}\right) \mathbf{v}\right] \cdot \dot{\mathbf{n}} d a \\
& +\int_{\mathcal{P}_{t}}\left(\mathbf{T}_{\mathrm{a}}-\varrho \frac{\partial \sigma}{\partial \mathbf{B}_{\mathrm{e}}}\right) \cdot \mathbf{B}_{\mathrm{e}}^{\nabla} d v
\end{aligned}
$$

where the Cauchy stress tensor and the molecular field are found to be (again for more details, see the Appendix)

$$
\begin{gathered}
\mathbf{T}=-\varrho^{2} \frac{\partial \sigma}{\partial \varrho} \mathbf{I}+2 \varrho \frac{\partial \sigma}{\partial \mathbf{B}_{\mathrm{e}}} \mathbf{B}_{\mathrm{e}}-\varrho(\nabla \mathbf{n})^{T} \frac{\partial \sigma}{\partial \boldsymbol{\nabla n}} \\
\mathbf{h}:=\varrho \frac{\partial \sigma}{\partial \mathbf{n}}-\operatorname{div}\left(\varrho \frac{\partial \sigma}{\partial \boldsymbol{\nabla} \mathbf{n}}\right) .
\end{gathered}
$$

\section{Governing equations}

According to the model, the material response is elastic with respect to the natural configuration. In other words, energy dissipation is uniquely associated to the evolution of the natural or stress-free configuration of the body, i.e., energy is dissipated only when microscopic reorganization occurs. As a consequence, only the term containing $\mathbf{B}_{\mathrm{e}}^{\nabla}$ in Eq. (9) yields a positive contribution to the dissipation, while the first four integrals must vanish. Given the arbitrariness of $\mathcal{P}_{t}$ and of the test fields, it is natural to use a generalized Rayleigh principle [36,37] and impose the vanishing of the corresponding generalized forces. In our case, this yields the usual balance of momentum equation

$$
\varrho \dot{\mathbf{v}}=\mathbf{b}+\operatorname{div} \mathbf{T}
$$

with boundary condition $\mathbf{t}_{(\boldsymbol{v})}=\mathbf{T} \boldsymbol{v}$. In particular, when the energy density (3) is substituted into Eq. (10), the Cauchy

\footnotetext{
${ }^{2}$ It is clear from Eq. (8) that $\mathbf{B}_{\mathrm{e}}^{\nabla}=0$ if and only if $\dot{\mathbf{H}}=0$. The tensor $\mathbf{H}=\left(\mathbf{G}^{T} \mathbf{G}\right)^{-1}$ is related to material remodeling and no remodeling occurs when the deformation is purely elastic.
} 
stress tensor reads

$$
\mathbf{T}=-p \mathbf{I}+\varrho \mu\left(\boldsymbol{\Psi}^{-1} \mathbf{B}_{\mathrm{e}}-\mathbf{I}\right)-\varrho(\nabla \mathbf{n})^{T} \frac{\partial \sigma}{\partial \boldsymbol{\nabla} \mathbf{n}},
$$

where the pressurelike function $p$ is

$$
p=\varrho^{2}\left[\frac{\partial \sigma_{0}}{\partial \varrho}-\mu \frac{3 a^{\prime}(\varrho)}{2 a(\varrho)}\left(\mathbf{n} \otimes \mathbf{n}-\frac{1}{3} \mathbf{I}\right) \cdot\left(\Psi^{-1} \mathbf{B}_{\mathrm{e}}\right)\right] .
$$

It is worth noticing that $\mathbf{T}$ does not explicitly contain any "active component." Activity is taken into account implicitly via the evolution equation for the natural configuration that describes how $\mathbf{B}_{\mathrm{e}}$ evolves in time. I shall describe this equation below.

To obtain an equation for the microstructure $\mathbf{n}$, we impose the vanishing of the third and fourth integrals in Eq. (9). Since $\mathbf{n} \cdot \dot{\mathbf{n}}=0$, it is sufficient to posit

$$
\mathbf{n} \times(\mathbf{g}-\mathbf{h})=0,
$$

with boundary condition

$$
\mathbf{n} \times \mathbf{m}_{(\boldsymbol{v})}=\mathbf{n} \times\left(\varrho \frac{\partial \sigma}{\partial \boldsymbol{\nabla} \mathbf{n}}\right) \boldsymbol{v} .
$$

These two last equations describe the evolution of the director field and are usually interpreted as balance of torques. Contrary to the first appearance, they do not describe a dissipationless motion; energy dissipation is again contained in their dependence on $\mathbf{B}_{\mathrm{e}}$. It is possible to show that, under the approximation of fast relaxation times, Eq. (15) reproduces exactly the usual director dynamics of liquid crystals, where the rotational Leslie coefficients $\alpha_{2}$ and $\alpha_{3}$ are identified in terms of our model parameters [27,30].

Finally, a positive dissipation for any $\mathcal{P}_{t}$ at all times implies

$$
\left(\mathbf{T}_{\mathrm{a}}-\varrho \frac{\partial \sigma}{\partial \mathbf{B}_{\mathrm{e}}}\right) \cdot \mathbf{B}_{\mathrm{e}}^{\nabla} \geqslant 0,
$$

where in our specific case

$$
\frac{\partial \sigma}{\partial \mathbf{B}_{\mathrm{e}}}=\frac{1}{2} \mu\left(\boldsymbol{\Psi}^{-1}-\mathbf{B}_{\mathrm{e}}^{-1}\right) .
$$

It is customary, when dealing with irreversible processes near equilibrium, to interpret the dissipation (or rate of entropy production) as the product of "fluxes" and "forces" and to assume a linear coupling between them. Furthermore, Onsager reciprocal relations impose additional symmetry constraints on this linear dependence. Hence, I assume that the evolution of the microscopic remodeling is governed by the following "gradient-flow" equation for $\mathbf{B}_{\mathrm{e}}$ :

$$
\mathbb{D}\left(\mathbf{B}_{\mathrm{e}}^{\nabla}\right)+\varrho \frac{\partial \sigma}{\partial \mathbf{B}_{\mathrm{e}}}=\mathbf{T}_{\mathrm{a}},
$$

where $\mathbb{D}$ is a fourth-rank tensor with the major symmetries. It is positive definite so that the dissipation inequality (17) is automatically satisfied. Moreover, I take it to be compatible with the underlying uniaxial symmetry along $\mathbf{n}$ of the microscopic constituents. The elements of the dissipation tensor $\mathbb{D}$ contain the characteristic relaxation times, and it is possible to show that there are only four relaxation times allowed by symmetry for rodlike constituents. I refer the interested reader to Ref. [30] for a more detailed discussion on these points.

\section{E. Interpretation of the active stress}

By contrast with standard theories, the present model does not explicitly include an active term in the Cauchy stress tensor. However, it is possible to recast our stress tensor, as given in Eq. (10), in a different form so that an analog of the "active stress" appears. To this end, I substitute Eq. (19) into Eq. (10) and obtain

$$
\mathbf{T}=-\varrho \frac{\partial \sigma}{\partial \varrho} \mathbf{I}+2\left[\mathbf{T}_{\mathrm{a}}-\mathbb{D}\left(\mathbf{B}_{\mathrm{e}}^{\nabla}\right)\right] \mathbf{B}_{\mathrm{e}}-\varrho(\nabla \mathbf{n})^{T} \frac{\partial \sigma}{\partial \nabla \mathbf{n}} .
$$

The active contribution now comes from the term $2 \mathbf{T}_{\mathrm{a}} \mathbf{B}_{\mathrm{e}}$ and it is not exactly equivalent to $\mathbf{T}_{\zeta}$ because it contains the effective strain tensor $\mathbf{B}_{\mathrm{e}}$. However, the analogy becomes more concrete if we make the approximation of small effective deformations, studied in the next section.

\section{SMALL EFFECTIVE DEFORMATIONS}

There are essentially two time scales in the problem. One characteristic time is dictated by the macroscopic deformation and is thus related to $\nabla \mathbf{v}$ in the following way:

$$
\tau_{\mathrm{def}}=1 /\|\nabla \mathbf{v}\| \text {. }
$$

The second characteristic time is connected to material remodeling and determines the time rate at which $\mathbf{B}_{\mathrm{e}}$ reaches its equilibrium value. I posit $\tau_{\text {rel }}=2\|\mathbb{D}\| / \varrho \mu$, where $\varrho \mu$ is a characteristic shear modulus.

We want to study the asymptotic approximation of the theory in the limit $\tau_{\text {rel }} \ll \tau_{\text {def }}$, that is,

$$
\tau_{\text {rel }}\|\nabla \mathbf{v}\|=\varepsilon \ll 1 .
$$

In this limit, reorganization is much faster than deformation and the theory reduces to a purely hydrodynamic theory, i.e., viscoelasticity becomes a higher order phenomenon and, to first order, viscosity coefficients are obtained as the product of the shear modulus and the relaxation times. An analysis of this approximation for the passive case is presented in Refs. [29,30]

For simplicity, I further assume that the active term only introduces a small perturbation of the passive dynamics so that the effective strain tensor $\mathbf{B}_{\mathrm{e}}$ is only a slight perturbation of its (passive) equilibrium value

$$
\mathbf{B}_{\mathrm{e}}=\boldsymbol{\Psi}+\mathbf{B}_{1}, \quad \text { with } \quad\left\|\mathbf{B}_{1}\right\|=O(\varepsilon),
$$

To leading order, the substitution of (22) into Eq. (20) yields the Cauchy stress tensor

$$
\mathbf{T}=-\varrho^{2} \frac{\partial \sigma}{\partial \varrho} \mathbf{I}-2 \mathbb{D}\left(\boldsymbol{\Psi}^{\nabla}\right) \boldsymbol{\Psi}+2 \mathbf{T}_{\mathrm{a}} \boldsymbol{\Psi}-\varrho(\boldsymbol{\nabla} \mathbf{n})^{T} \frac{\partial \sigma}{\partial \boldsymbol{\nabla} \mathbf{n}} .
$$

The codeformational derivative of the shape tensor reads

$$
\begin{aligned}
& \boldsymbol{\Psi}^{\nabla}=\frac{\varrho a^{\prime}(\varrho)}{a(\varrho)^{2}}(\operatorname{tr} \mathbf{D})\left\{\mathbf{I}-\left[1+2 a(\varrho)^{3}\right](\mathbf{n} \otimes \mathbf{n})\right\}
\end{aligned}
$$

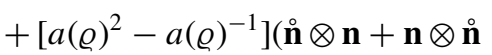

$$
\begin{aligned}
& -\mathbf{D n} \otimes \mathbf{n}-\mathbf{n} \otimes \mathbf{D n})-2 a(\varrho)^{-1} \mathbf{D},
\end{aligned}
$$

where $\stackrel{\circ}{\mathbf{n}}=\dot{\mathbf{n}}-\mathbf{W n}, \mathbf{W}=\left(\nabla \mathbf{v}-\nabla \mathbf{v}^{T}\right) / 2$ is the spin tensor and $\mathbf{D}=\left(\nabla \mathbf{v}+\nabla \mathbf{v}^{T}\right) / 2$ is the stretching tensor.

The first two terms in Eq. (23) reproduce the passive dynamics of nematic liquid crystals. Indeed, they reduce 
exactly to the Cauchy stress tensor as given in the classical (compressible) Ericksen-Leslie theory [32]

$$
\begin{aligned}
& \mathbf{T}_{\mathrm{EL}}=-p \mathbf{I}+\alpha_{1}(\mathbf{n} \cdot \mathbf{D n})(\mathbf{n} \otimes \mathbf{n})+\alpha_{2}(\mathbf{n} \otimes \mathbf{n})+\alpha_{3}(\mathbf{n} \otimes \stackrel{\mathbf{n}}{)} \\
& +\alpha_{4} \mathbf{D}+\alpha_{5}(\mathbf{D n} \otimes \mathbf{n})+\alpha_{6}(\mathbf{n} \otimes \mathbf{D n}) \\
& +\alpha_{7}[(\operatorname{tr} \mathbf{D})(\mathbf{n} \otimes \mathbf{n})+(\mathbf{n} \cdot \mathbf{D n}) \mathbf{I}]+\alpha_{8}(\operatorname{tr} \mathbf{D}) \mathbf{I} \text {, }
\end{aligned}
$$

after the viscosity coefficients $\alpha_{1}, \ldots, \alpha_{8}$ have been suitably identified. An explicit comparison is carried out in [30].

The third term, $2 \mathbf{T}_{\mathrm{a}} \boldsymbol{\Psi}$, corresponds to an active stress and as such can be compared with the active stress $\mathbf{T}_{\zeta} \propto \mathbf{Q}$, as given in the usual theories $[3,4,6-8,38]$. With the simple choice

$$
\mathbf{T}_{\mathrm{a}}=-\frac{1}{2} \varrho \mu \zeta \mathbf{I},
$$

where $\zeta$ is a dimensionless modeling parameter that controls the activity of the body, $2 \mathbf{T}_{\mathrm{a}} \boldsymbol{\Psi}$ shares the same uniaxial symmetry of $\mathbf{T}_{\zeta}$ and takes the form of a dipole interaction. Other choices are of course possible and the specific form of $\mathbf{T}_{\mathrm{a}}$ has to be inferred by the interaction of a number of features, namely, the properties of the particular material under study, the experimental results, and microscopic theoretical investigations. Instead of an isotropic $\mathbf{T}_{\mathbf{a}}$, a second natural possibility is to choose $\mathbf{T}_{\mathrm{a}} \propto \boldsymbol{\Psi}$. However, in both cases the resulting active stress, $2 \mathbf{T}_{\mathrm{a}} \boldsymbol{\Psi}$, possesses the same uniaxial symmetry about the director $\mathbf{n}$. Therefore, both choices should lead to essentially the same qualitative results.

Finally, the last term in Eq. (23) is standard and it is a consequence of the elastic distortions of the director field.

\section{SPONTANEOUS FLOW AND SELF-CHANNELING}

A key prediction of continuum models of active liquid crystals is the existence of spontaneously generated fluid flows in one-dimensional channels $[11,12,38,39]$. It is then natural to test the present model against such predictions. To this end, I analyze the hydrodynamic equations in the simple geometry of a two-dimensional channel of infinite length along the $x$ direction and with height $L$ (see Fig. 1).

For simplicity, I also assume that there is only one relaxation time, i.e., $\mathbb{D}=\frac{1}{2} \varrho \mu \tau \mathbb{I}$, and that $\tau$ is much smaller than the characteristic times of the flow (I is the fourth-rank identity tensor). Hence, it is possible to use the approximation of small effective deformations, as developed in Sec. III, where the Cauchy stress tensor is given as in Eq. (23). I also assume incompressibility so that the pressure is now

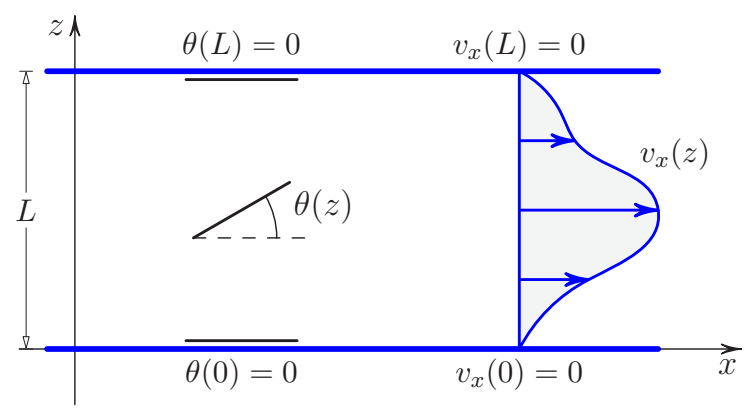

FIG. 1. Schematic representation of the channel geometry studied in the text. a Lagrange multiplier. I consider a two-dimensional active nematic suspension with uniform degree of orientation and discuss the spatial dependence of the fields $\theta(z)$ (the angle that the nematic units form with the $x$ axis) and $v_{x}(z)$ (the $x$ component of the macroscopic velocity of the suspension). Both fields depend only on $z$ because of the translational invariance along $x$. I assume no-slip boundary conditions at both surfaces and $\theta(0)=\theta(L)=0$. The equations of motion in steady conditions are the Stokes equations $\partial T_{x z} / \partial z=0$, $\partial T_{z z} / \partial z=0$ and the director equation $\mathbf{n} \times \mathbf{h}=0$, namely, Eq. (15) with $\mathbf{g}=0$. The second of these equations determines the pressure in the film, and I will not discuss this in more detail. More interesting are the first and the third that read (see Appendix B for an explicit derivation)

$$
\begin{aligned}
& 4\left(a_{0}^{3}-1\right) \theta^{\prime}\left\{2 \tau v_{x}^{\prime} \sin (2 \theta)\left[\left(a_{0}^{3}-1\right) \cos (2 \theta)+a_{0}^{3}+1\right]\right. \\
& \left.-2 a_{0} \zeta \cos (2 \theta)\right\}-\tau v_{x}^{\prime \prime}\left[4\left(a_{0}^{6}-1\right) \cos (2 \theta)-5 a_{0}^{6}\right. \\
& \left.+\left(a_{0}^{3}-1\right)^{2} \cos (4 \theta)+2 a_{0}^{3}-5\right]=0, \\
& \left(a_{0}^{3}-1\right) \mu \tau v_{x}^{\prime}\left[\left(a_{0}^{3}+1\right) \cos (2 \theta)-a_{0}^{3}+1\right]+2 a_{0}^{2} k \theta^{\prime \prime}=0,
\end{aligned}
$$

with boundary conditions $\theta(0)=\theta(L)=0$ and $v_{x}(0)=$ $v_{x}(L)=0$. The coefficient $a_{0}$ is the shape parameter that identifies the form of the shape tensor at the given density $\varrho_{0}$ : $a_{0}=a\left(\varrho_{0}\right)$. The Oseen-Frank potential, $\sigma_{\mathrm{Fr}}(\varrho, \mathbf{n}, \nabla \mathbf{n})$, is simply taken to be $\sigma_{\mathrm{Fr}}=k|\nabla \mathbf{n}|^{2}$ (one-constant approximation).

It is straightforward to check that $\theta(z)=0$ and $v_{x}(z)=0$ is always a solution of (27), for any value of the parameters. However, above a critical threshold for the thickness $L$, a bifurcation occurs and the trivial solution is no longer unique. The critical condition is obtained by performing a linear stability analysis. The linearized equations about the trivial solution read

$$
\begin{aligned}
& \tau v_{x}^{\prime \prime}(z)-\zeta a_{0}\left(a_{0}^{3}-1\right) \theta^{\prime}(z)=0, \\
& a_{0}^{2} k \theta^{\prime \prime}(z)+\mu \tau\left(a_{0}^{3}-1\right) v_{x}^{\prime}(z)=0 .
\end{aligned}
$$

It is known $[11,12,38]$ that both the polar and the apolar systems exhibit a pitchfork bifurcation, i.e., a Freédericksz-like transition, between a state where the director field is constant and parallel to the walls throughout the channel to a nonuniformly oriented state in which the system spontaneously flows in the $x$ direction. The transition can be tuned by changing either the film thickness or the activity parameter.

However, in our case, the linearized equations (28) admit two independent modes of instability at the bifurcation and hence (28) show a more complex behavior than a simple pitchfork bifurcation. Namely, the critical condition reads

$$
L \sqrt{\frac{\left(a_{0}^{3}-1\right)^{2}}{a_{0}} \frac{\mu \zeta}{k}}=2 \pi n,
$$

where $n$ is an integer. For a given $L$, the lowest critical value of the activity, $\zeta_{c}$, is the one where bifurcation occurs and corresponds to the fundamental mode $n=1$. Likewise, for a given activity coefficient $\zeta$, it is possible to define a critical length $L_{c}$ corresponding to the solution of (29) with $n=1$. It is interesting to observe that $L_{c}$ diverges to infinity, and no 


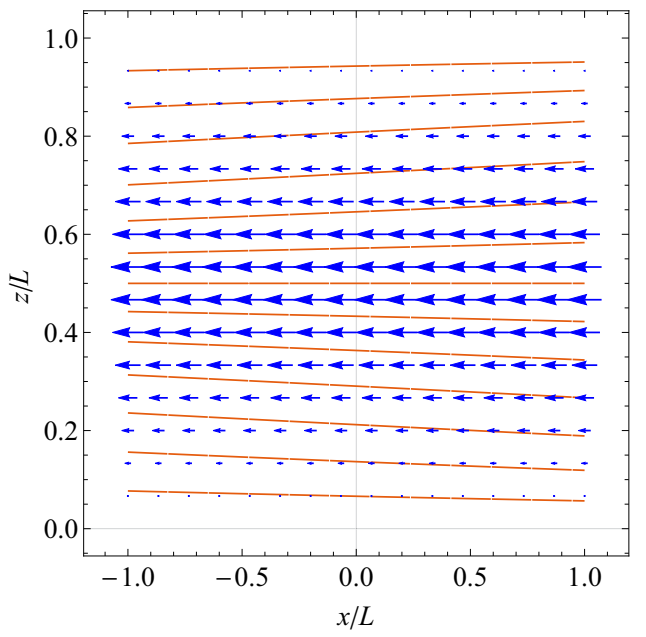

FIG. 2. Spontaneous flow. Vector plot of the solutions of Eqs. (27), with $a_{0}=2.5, \zeta=0.5$, and $L=1.1 L_{c}$, where $L_{c}$ is given as in Eq. (29) $(n=1)$. Blue arrows represent the velocity field $v_{x}(z)$, while orange lines depict the director field.

bifurcation occurs, either for $\zeta \rightarrow 0$ (passive case) or $a_{0} \rightarrow$ 1 (isotropic case). When the condition (29) is satisfied, the nontrivial solutions of Eqs. (28) are

$$
\begin{aligned}
\theta(z) & =C_{1} \sin ^{2}\left(\frac{\pi n z}{L}\right)+C_{2} \sin \left(\frac{2 \pi n z}{L}\right), \\
v_{x}(z) & =\frac{a_{0}\left(a_{0}^{3}-1\right) \zeta L}{4 \pi n \tau}\left[4 C_{2} \sin ^{2}\left(\frac{\pi n z}{L}\right)-C_{1} \sin \left(\frac{2 \pi n z}{L}\right)\right],
\end{aligned}
$$

with $C_{1}$ and $C_{2}$ arbitrary coefficients. Therefore, they span a two-dimensional linear space for each value of $n>0$.

This twofold instability is confirmed by a numerical analysis. In the numerical code, $L$ is used to set the length scale, while $\tau$ sets the time scale. The nondimensional parameters $a_{0}$ and $\zeta$ are fixed to be equal to 2.5 and 0.5 , respectively. We then perform the numerical integration of the nonlinear equations (27), where the ratio $k / \mu$ is chosen such that the critical length $L_{c}$ can be suitably adjusted.

Figure 2 shows a numerical solution of Eqs. (27) for $L=1.1 L_{c}$. The first mode to be excited above the bifurcation is the "spontaneous flow" mode, corresponding to $C_{1}=0$ and $C_{2} \neq 0$ in Eqs. (30). A right or left steady flow of active particles is spontaneously generated due to activity. Upon further increasing $L / L_{c}$, also the self-channeling mode, corresponding to $C_{1} \neq 0$ and $C_{2}=0$, appears. Figure 3 shows a numerical solution of Eqs. (27) with $L=1.4 L_{c}$. Here, the active particles self-organize into subchannels and show no net flow of particles.

While a Freédericksz-like transition from a planar motionless state to a flowing state has been predicted for increasing thickness, in an active nematic gel the transition to a selfchanneling state seems to be new. A similar effect has been described in [38], but in the case of a two-dimensional square domain with periodic boundary conditions. The authors of Ref. [38] do not observe any banding in the simple onedimensional geometry considered here. By contrast, active

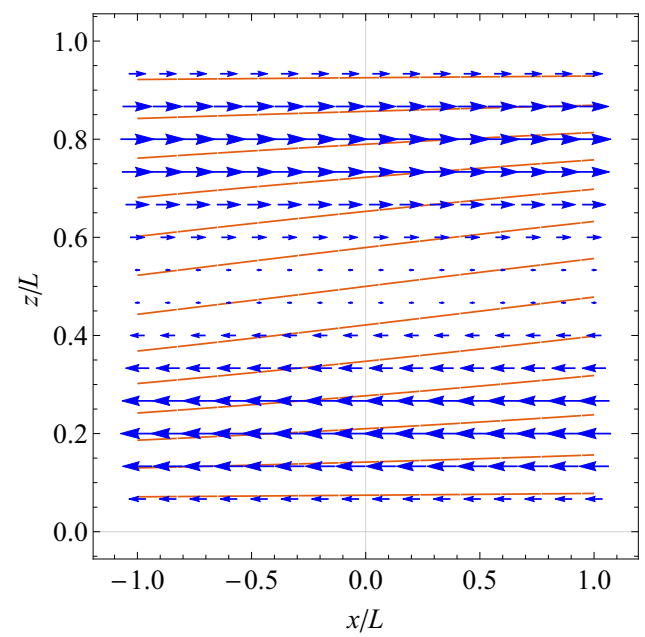

FIG. 3. Self-channeling. Vector plot of the solutions of Eqs. (27), with $a_{0}=2.5, \zeta=0.5$, and $L=1.4 L_{c}$, where $L_{c}$ is given as in Eq. (29) $(n=1)$. Blue arrows represent the velocity field $v_{x}(z)$, while orange lines depict the director field.

polar gels seem to show a richer behavior where selfchanneling appears [11,12].

\section{CONCLUSION}

Active nematic gels are nonconventional materials that mimic the behavior of living matter. They represent an excellent playground for a deeper understanding of the mechanical response of biological tissues such as the cellular cytoskeleton, a network of cross-linked filaments subjected to the action of molecular motors.

The simplest theory of active nematic gels takes inspiration from liquid-crystal theory, which is then typically supplemented by adding an active stress and a viscoelastic relaxation time to the passive theory. However, this model does not explicitly take into account the fact that molecular motors act at the microscopic level by modifying the way in which the material reorganizes its internal structure.

To this end, I have put forth a thermodynamically consistent theory of active nematic gels that naturally embeds viscoelasticity and introduces activity as a remodeling force so that no active stress has to be added to the model. The active remodeling force competes with the natural relaxation process of the passive systems and drives the system out of equilibrium.

Finally, in order to explore the early consequences of the theory, I have studied the dynamical properties of thin films of active nematic fluids. Above a critical thickness of the film a rich variety of complex behaviors is observed. Namely, at the same critical length, I find both a spontaneous flow of active particles and a self-channelling effect, where the particles organize themselves into sub-channels and flow in opposite directions.

\section{ACKNOWLEDGMENTS}

I wish to thank Davide Ambrosi and Antonio Di Carlo for their encouragement and for stimulating discussions. 


\section{APPENDIX A: DERIVATION OF THE CAUCHY STRESS TENSOR AND THE MOLECULAR FIELD}

We need two simple lemmas, which I state without proofs.

Lemma 1. Let $f$ be a function depending only on $\mathbf{F}_{\mathrm{e}}$ (or $\left.\mathbf{B}_{\mathrm{e}}\right)$. Then,
(1) $\frac{\partial f}{\partial \mathbf{F}} \mathbf{F}^{T}=\frac{\partial f}{\partial \mathbf{F}_{\mathrm{e}}} \mathbf{F}_{\mathrm{e}}^{T}=2 \frac{\partial f}{\partial \mathbf{B}_{\mathrm{e}}} \mathbf{B}_{\mathrm{e}}$.
(2) $\mathbf{F}^{-T} \frac{\partial f}{\partial \mathbf{H}} \mathbf{F}^{-1}=\frac{\partial f}{\partial \mathbf{B}_{\mathrm{e}}}$.

Lemma 2. Let $f=f(\varrho)$ be a function of $\varrho$ only. Then, $\frac{\partial f}{\partial \mathbf{F}} \mathbf{F}^{T}=-\varrho \frac{\partial f(\varrho)}{\partial \varrho} \mathbf{I}$.

We can now calculate the material derivative of $\mathcal{F}$ :

$\dot{\mathcal{F}}=\int_{\mathcal{P}_{t}}\left[\varrho \frac{\partial \sigma}{\partial \mathbf{F}} \cdot \dot{\mathbf{F}}+\varrho \frac{\partial \sigma}{\partial \mathbf{H}} \cdot \dot{\mathbf{H}}+\varrho \frac{\partial \sigma}{\partial \mathbf{n}} \cdot \dot{\mathbf{n}}+\varrho \frac{\partial \sigma}{\partial \nabla \mathbf{n}} \cdot(\nabla \mathbf{n}) \cdot\right] d v$,

which is then simplified with the use of Lemma 1 and the identities

$$
\begin{gathered}
\dot{\mathbf{F}}=(\nabla \mathbf{v}) \mathbf{F}, \\
\frac{D}{D t}(\nabla \mathbf{n})=\nabla \dot{\mathbf{n}}-(\nabla \mathbf{n})(\nabla \mathbf{v}), \\
\dot{\mathbf{H}}_{\mathbf{F}^{T}=\left(\mathbf{B}_{\mathrm{e}}\right)} \cdot-(\nabla \mathbf{v}) \mathbf{B}_{\mathrm{e}}-\mathbf{B}_{\mathrm{e}}(\nabla \mathbf{v})^{T}=: \mathbf{B}_{\mathrm{e}}^{\nabla},
\end{gathered}
$$

so that the rate of change of the free energy reads

$$
\begin{aligned}
\dot{\mathcal{F}}= & \int_{\mathcal{P}_{t}}\left(\varrho \frac{\partial \sigma}{\partial \mathbf{F}} \mathbf{F}^{T}-\varrho(\nabla \mathbf{n})^{T} \frac{\partial \sigma}{\partial \boldsymbol{\nabla n}}\right) \cdot \nabla \mathbf{v} d v \\
& +\int_{\mathcal{P}_{t}}\left(\varrho \frac{\partial \sigma}{\partial \mathbf{n}}+\varrho \frac{\partial \sigma}{\partial \nabla \mathbf{n}} \cdot \nabla \dot{\mathbf{n}}\right) d v+\int_{\mathcal{P}_{t}} \varrho \frac{\partial \sigma}{\partial \mathbf{B}_{\mathrm{e}}} \cdot \mathbf{B}_{\mathrm{e}}^{\nabla} d v .
\end{aligned}
$$

Further simplifications are obtained by employing the divergence theorem in the first two integrals

$$
\begin{aligned}
& \int_{\mathcal{P}_{t}} \mathbf{X} \cdot \nabla \mathbf{v} d v=\int_{\partial \mathcal{P}_{t}} \mathbf{X} \boldsymbol{v} \cdot \mathbf{v} d a-\int_{\mathcal{P}_{t}} \operatorname{div}(\mathbf{X}) \cdot \mathbf{v} d v \\
& \int_{\mathcal{P}_{t}} \mathbf{X} \cdot \nabla \dot{\mathbf{n}} d v=\int_{\partial \mathcal{P}_{t}} \mathbf{X v} \cdot \dot{\mathbf{n}} d a-\int_{\mathcal{P}_{t}} \operatorname{div}(\mathbf{X}) \cdot \dot{\mathbf{n}} d v,
\end{aligned}
$$

where $\mathbf{X}$ is a generic second-rank tensor. In so doing, we recognize the generalized forces paired to the fields $\mathbf{v}$ and $\dot{\mathbf{n}}$ and obtain

$$
\begin{aligned}
\dot{\mathcal{F}}= & \int_{\partial \mathcal{P}_{t}}\left(\varrho \frac{\partial \sigma}{\partial \mathbf{F}} \mathbf{F}^{T}-\varrho(\nabla \mathbf{n})^{T} \frac{\partial \sigma}{\partial \nabla \mathbf{n}}\right) \boldsymbol{v} \cdot \mathbf{v} d a \\
& -\int_{\mathcal{P}_{t}} \operatorname{div}\left(\varrho \frac{\partial \sigma}{\partial \mathbf{F}} \mathbf{F}^{T}-\varrho(\nabla \mathbf{n})^{T} \frac{\partial \sigma}{\partial \boldsymbol{\nabla n}}\right) \cdot \mathbf{v} d v \\
& +\int_{\partial \mathcal{P}_{t}}\left(\varrho \frac{\partial \sigma}{\partial \nabla \mathbf{n}}\right) \boldsymbol{v} \cdot \dot{\mathbf{n}} d a \\
& +\int_{\mathcal{P}_{t}}\left[\varrho \frac{\partial \sigma}{\partial \mathbf{n}}-\operatorname{div}\left(\varrho \frac{\partial \sigma}{\partial \boldsymbol{\nabla n}}\right)\right] \cdot \dot{\mathbf{n}} d v \\
& +\int_{\mathcal{P}_{t}} \varrho \frac{\partial \sigma}{\partial \mathbf{B}_{\mathrm{e}}} \cdot \mathbf{B}_{\mathrm{e}}^{\nabla} d v
\end{aligned}
$$

It is then natural to define the Cauchy stress tensor (conjugate to $\mathbf{v}$ ) and the molecular field (conjugate to $\dot{\mathbf{n}}$ )

$$
\begin{aligned}
\mathbf{T} & :=\varrho \frac{\partial \sigma}{\partial \mathbf{F}} \mathbf{F}^{T}-\varrho(\boldsymbol{\nabla n})^{T} \frac{\partial \sigma}{\partial \boldsymbol{\nabla} \mathbf{n}}, \\
\mathbf{h} & :=\varrho \frac{\partial \sigma}{\partial \mathbf{n}}-\operatorname{div}\left(\varrho \frac{\partial \sigma}{\partial \boldsymbol{\nabla} \mathbf{n}}\right) .
\end{aligned}
$$

A further application of Lemmas 1 and 2 allow us to rewrite the stress tensor (A9) as in Eq. (10).

\section{APPENDIX B: DERIVATION OF EQ. (27)}

Let us denote with $\left\{\mathbf{e}_{x}, \mathbf{e}_{y}, \mathbf{e}_{z}\right\}$ the Cartesian unit vectors along the coordinate axes. We posit a stationary velocity field of the form $\mathbf{v}=v_{x}(z) \mathbf{e}_{x}$. Hence, the gradient of velocity is $\nabla \mathbf{v}=v_{x}^{\prime}(z) \mathbf{e}_{x} \otimes \mathbf{e}_{z}$, and the material time derivative of $\mathbf{v}$ vanishes. The director field is described by the angle $\theta(z)$ such that $\mathbf{n}=\cos \theta(z) \mathbf{e}_{x}+\sin \theta(z) \mathbf{e}_{z}$. It is also useful to introduce the orthogonal unit vector $\mathbf{n}^{\perp}=-\sin \theta(z) \mathbf{e}_{x}+\cos \theta(z) \mathbf{e}_{z}$. With the simplifying assumptions

$$
\mathbf{T}_{\mathrm{a}}=-\frac{1}{2} \varrho \mu \zeta \mathbf{I}, \quad \mathbb{D}=\frac{1}{2} \varrho \mu \tau \mathbb{I},
$$

the stress tensor, as given in Eq. (23), reads

$$
\mathbf{T}=-p \mathbf{I}-\varrho \mu\left(\tau \boldsymbol{\Psi}^{\nabla} \boldsymbol{\Psi}+\zeta \boldsymbol{\Psi}\right)-\varrho k(\nabla \mathbf{n})^{T}(\nabla \mathbf{n}),
$$

where $\nabla \mathbf{n}=\theta^{\prime}(z) \mathbf{n}^{\perp} \otimes \mathbf{e}_{z}$, so that

$$
(\nabla \mathbf{n})^{T}(\nabla \mathbf{n})=\theta^{\prime}(z)^{2} \mathbf{e}_{z} \otimes \mathbf{e}_{z}
$$

To calculate the codeformational derivative of the shape tensor, we observe that $\frac{\partial \Psi}{\partial t}=0$ and $(\nabla \boldsymbol{\Psi}) \mathbf{v}=0$ so that we obtain

$$
\begin{aligned}
\boldsymbol{\Psi}^{\nabla}= & \frac{\partial \boldsymbol{\Psi}}{\partial t}+(\nabla \boldsymbol{\Psi}) \mathbf{v}-(\boldsymbol{\nabla} \mathbf{v}) \boldsymbol{\Psi}-\boldsymbol{\Psi}\left(\boldsymbol{\nabla} \mathbf{v}^{T}\right) \\
= & -\left(a_{0}^{2}-\frac{1}{a_{0}}\right) v_{x}^{\prime}(z) \sin \theta(z)\left(\mathbf{e}_{x} \otimes \mathbf{n}+\mathbf{n} \otimes \mathbf{e}_{x}\right) \\
& -\frac{1}{a_{0}} v_{x}^{\prime}(z)\left(\mathbf{e}_{x} \otimes \mathbf{e}_{z}+\mathbf{e}_{z} \otimes \mathbf{e}_{x}\right)
\end{aligned}
$$

where we have assumed incompressibility with constant density $\varrho_{0}$ and $a\left(\varrho_{0}\right)=a_{0}$, so that the pressure $p$ is a Lagrange multiplier. This derivative is then inserted into Eq. (B2) to obtain, with lengthy but straightforward calculations, the Cauchy stress tensor.

Since all the terms depend only on the variable $z$, the Stokes equations reduce to

$$
\frac{\partial T_{x z}}{\partial z}=0, \quad \frac{\partial T_{z z}}{\partial z}=0 .
$$

The latter yields an equation for the pressure, while the former is

$$
\begin{aligned}
& \frac{d}{d z}\left\{-\frac{\varrho \mu}{8 a_{0}^{2}}\left[4 a_{0}\left(a_{0}^{3}-1\right) \zeta \sin [2 \theta(z)]\right.\right. \\
& +\tau v_{x}^{\prime}(z)\left\{4\left(a_{0}^{6}-1\right) \cos [2 \theta(z)]-5 a_{0}^{6}\right. \\
& \left.\left.\left.+\left(a_{0}^{3}-1\right)^{2} \cos [4 \theta(z)]+2 a_{0}^{3}-5\right\}\right]\right\}=0
\end{aligned}
$$

and corresponds to Eq. (27a).

The evolution equation of the director furnishes Eq. (27b). In order to show this, let us calculate the molecular field $\mathbf{h}$, as 
given in Eq. (11). To this end, we observe that

$$
\begin{gathered}
\frac{\partial \sigma}{\partial \mathbf{n}}=2\left(a_{0}^{2}-a_{0}^{-1}\right) \frac{\partial \sigma}{\partial \boldsymbol{\Psi}} \mathbf{n}, \\
\frac{\partial}{\partial \boldsymbol{\Psi}} \operatorname{tr}\left(\boldsymbol{\Psi}^{-1} \mathbf{B}_{\mathrm{e}}\right)=-\boldsymbol{\Psi}^{-1} \mathbf{B}_{\mathrm{e}} \boldsymbol{\Psi}^{-1}, \\
\frac{\partial \sigma}{\partial \mathbf{n}}=-\mu\left(1-a_{0}^{-3}\right) \boldsymbol{\Psi}^{-1} \mathbf{B}_{\mathrm{e}} \mathbf{n}, \\
\operatorname{div} \frac{\partial \sigma}{\partial \boldsymbol{\nabla} \mathbf{n}}=k \operatorname{div} \boldsymbol{\nabla} \mathbf{n}=k\left(\theta^{\prime \prime} \mathbf{n}^{\perp}-\theta^{\prime 2} \mathbf{n}\right) .
\end{gathered}
$$

In agreement with Eq. (B2), the term $\Psi^{-1} \mathbf{B}_{\mathrm{e}}$ is

$$
\boldsymbol{\Psi}^{-1} \mathbf{B}_{\mathrm{e}}=\mathbf{I}-\tau \boldsymbol{\Psi}^{\nabla} \boldsymbol{\Psi}-\zeta \boldsymbol{\Psi}
$$

and the director equation, $\mathbf{n} \times \mathbf{h}=0$, then reads

$$
\mathbf{n} \times\left[\mu \tau\left(a_{0}^{2}-a_{0}^{-1}\right) \Psi^{\nabla} \mathbf{n}-k \theta^{\prime \prime} \mathbf{n}^{\perp}\right]=0 .
$$

After a further simplification, this coincides with Eq. (27b).
[1] A. W. C. Lau and T. C. Lubensky, Phys. Rev. E 80, 011917 (2009).

[2] F. Jülicher, K. Kruse, J. Prost, and J.-F. Joanny, Phys. Rep. 449, 3 (2007).

[3] J. Prost, F. Jülicher, and J. F. Joanny, Nat. Phys. 11, 111 (2015).

[4] M. C. Marchetti, J. F. Joanny, S. Ramaswamy, T. B. Liverpool, J. Prost, M. Rao, and R. A. Simha, Rev. Mod. Phys. 85, 1143 (2013).

[5] E. J. Hemingway, A. Maitra, S. Banerjee, M. C. Marchetti, S. Ramaswamy, S. M. Fielding, and M. E. Cates, Phys. Rev. Lett. 114, 098302 (2015).

[6] E. J. Hemingway, M. E. Cates, and S. M. Fielding, Phys. Rev. E 93, 032702 (2016).

[7] R. A. Simha and S. Ramaswamy, Phys. Rev. Lett. 89, 058101 (2002).

[8] S. P. Thampi, A. Doostmohammadi, R. Golestanian, and J. M. Yeomans, Europhys. Lett. 112, 28004 (2015).

[9] K. Kruse, J.-F. Joanny, F. Jülicher, J. Prost, and K. Sekimoto, Eur. Phys. J. E 16, 5 (2005).

[10] X. Yang, J. Li, M. G. Forest, and Q. Wang, Entropy 18, 202 (2016).

[11] R. Voituriez, J.-F. Joanny, and J. Prost, Europhys. Lett. 70, 404 (2005).

[12] S. A. Edwards and J. M. Yeomans, Europhys. Lett. 85, 18008 (2009).

[13] M. Ben Amar, O. V. Manyuhina, and G. Napoli, Eur. Phys. J. Plus 126, 19 (2011).

[14] E. Tjhung, D. Marenduzzo, and M. E. Cates, Proc. Natl. Acad. Sci. USA 109, 12381 (2012).

[15] L. Giomi and A. DeSimone, Phys. Rev. Lett. 112, 147802 (2014).

[16] A. DiCarlo, in Mechanics of Material Forces, Surface and Bulk Growth Unified, edited by P. Steinmann and G. A. Maugin (Springer, New York, 2005), Chap. 6, pp. 56-64.

[17] D. Ambrosi and F. Guana, Math. Mech. Solids 12, 319 (2007).

[18] J. Stålhand, A. Klarbring, and G. A. Holzapfel, Prog. Biophys. Mol. Biol. 96, 465 (2008).
[19] C. Verdier, J. Etienne, A. Duperray, and L. Preziosi, C. R. Phys. 10, 790 (2009).

[20] D. Ambrosi, L. Preziosi, and G. Z. Vitale, Angew Math. Phys. 61, 177 (2010).

[21] D. Ambrosi and S. Pezzuto, J. Elast. 107, 199 (2012).

[22] R. De Vita, R. Grange, P. Nardinocchi, and L. Teresi, J. Theor. Biol. 425, 1 (2017).

[23] H. R. Brand, H. Pleiner, and D. Svenšek, Eur. Phys. J. E 37, 83 (2014).

[24] E. Kröner, Arch. Ration. Mech. Anal. 4, 273 (1959).

[25] E. H. Lee, J. Appl. Mech. 36, 1 (1969).

[26] E. K. Rodriguez, A. Hoger, and A. D. McCulloch, J. Biomech. 27, 455 (1994)

[27] P. Biscari, A. DiCarlo, and S. S. Turzi, Soft Matter 10, 8296 (2014).

[28] S. S. Turzi, Eur. J. Appl. Math. 26, 93 (2015).

[29] P. Biscari, A. DiCarlo, and S. S. Turzi, Phys. Rev. E 93, 052704 (2016).

[30] S. S. Turzi, Phys. Rev. E 94, 062705 (2016).

[31] M. Warner and E. M. Terentjev, Liquid Crystal Elastomers, International Series of Monographs on Physics (Oxford University Press, Oxford, 2003).

[32] P. de Gennes and J. Prost, The Physics of Liquid Crystals, 2nd ed. (Oxford University Press, Oxford, 1995).

[33] D. D. Joseph, Fluid Dynamics of Viscoelastic Liquids (Springer, New York, 2013), Vol. 84.

[34] M. E. Gurtin, E. Fried, and L. Anand, The Mechanics and Thermodynamics of Continua (Cambridge University Press, Cambridge, 2010).

[35] A. DiCarlo and S. Quiligotti, Mech. Res. Commun. 29, 449 (2002).

[36] A. M. Sonnet and E. G. Virga, Phys. Rev. E 64, 031705 (2001).

[37] A. M. Sonnet and E. G. Virga, Dissipative Ordered Fluids: Theories for Liquid Crystals (Springer, New York, 2012).

[38] L. Giomi, L. Mahadevan, B. Chakraborty, and M. F. Hagan, Nonlinearity 25, 2245 (2012).

[39] L. Giomi, M. C. Marchetti, and T. B. Liverpool, Phys. Rev. Lett. 101, 198101 (2008). 